\title{
RINGS WITH A BOUNDED NUMBER OF GENERATORS FOR RIGHT IDEALS
}

\author{
WILLIAM D. BLAIR
}

\begin{abstract}
Let the ring $S$ be a finitely generated module over a subring $R$ of its center. Then it will be shown that $S$ has the property that every right ideal can be generated by a bounded number of elements if and only if $R$ has the property that every ideal can be generated by a bounded number of elements. As a corollary we show that a two-sided Noetherian affine ring satisfying a polynomial identity has the property that every right ideal can be generated by a bounded number of elements if and only if every left ideal can be generated by a bounded number of elements.
\end{abstract}

1. Introduction. Several authors have studied commutative Noetherian rings with the property that there exists an integer $k$ such that every ideal can be generated by $k$ or fewer elements. I. S. Cohen [3] showed that the classical Krull dimension of such rings is at most one, and that if $R$ is a local Noetherian domain of Krull dimension one then there exists a positive integer $k$ such that every ideal can be generated by $k$ or fewer elements. In the noncommutative case J. T. Stafford [11] has shown that if $R$ is a right and left Noetherian ring for which there exists a positive integer $k$ such that every right ideal of $R$ can be generated by $k$ or fewer elements then $R$ has classical Krull dimension at most one. An example of A. V. Jategaonkar [5] shows that the assumption that the ring is merely right Noetherian is not sufficient for Stafford's theorem.

J. D. Sally [9] calls a commutative ring bounded if there is a bound for the number of generators of all ideals. Using multiplicity theory she shows that if $R \subseteq S$ are commutative rings and if $S$ is a finitely generated $R$-module, then $R$ is bounded if and only if $S$ is bounded. In this paper we will show that this theorem can be extended to the case where $S$ is noncommutative and $R$ is central in $S$. Our proof provides a new approach to the commutative case since we avoid the use of multiplicity theory. As an application we apply our theorem to affine rings satisfying a polynomial identity. Finally, we discuss several examples which indicate obstructions to some natural generalizations of our results.

2. Preliminaries. All rings will be assumed to have a multiplicative identity element, and all subrings of a ring will have the same identity. $Z(R)$ will denote the center of the ring $R$.

Let $R$ be a ring and $M$ a right $R$-module. If every right (resp. left) ideal of $R$ can be generated by $k$ or fewer elements we say that $R$ satisfies $r$-Bnd $(k)$ (resp. $l$-Bnd $(k))$. In the commutative case we omit the $r$ or $l$. We say that $R$ satisfies $r$-Bnd (resp. $l$-Bnd) if $R$ satisfies $r$-Bnd $(k)$ (resp. $l$-Bnd $(k)$ ) for some $k$. If every

Received by the editors September 9, 1985.

1980 Mathematics Subject Classification. Primary 16A33, 16A38. 
submodule of $M$ can be generated by $k$ or fewer elements we say that $M$ satisfies $r-\operatorname{Bnd}(k)$,

If every chain of prime ideals has at most two distinct elements, we say that the classical Krull dimension of $R$ is at most one. Since we will not need to mention other Krull dimensions we omit the adjective classical from now on.

LEMMA 1. Let $N \subseteq M$ be right $R$-modules.

(i) If $M$ satisfies $r$ - $\operatorname{Bnd}(k)$ then $N$ and $M / N$ satisfy $r-\operatorname{Bnd}(k)$.

(ii) If $N$ satisfies $r$ - $\operatorname{Bnd}\left(k_{1}\right)$ and $M / N$ satisfies $r-\operatorname{Bnd}\left(k_{2}\right)$, then $M$ satisfies $r-\operatorname{Bnd}\left(k_{1}+k_{2}\right)$.

(iii) If $M=m_{1} R+m_{2} R+\cdots+m_{t} R$ is a finitely generated right $R$-module and $R$ satisfies $r-\operatorname{Bnd}(k)$, then $M$ satisfies $r$ - $\operatorname{Bnd}(k \cdot t)$.

ProOF. (i) and (ii) are easy, and (iii) follows from (i) and (ii) by induction.

LEMMA 2. (i) If the ring $R_{i}$ satisfies $r$-Bnd $\left(k_{i}\right)$ for $i=1,2, \ldots, t$, then $R=$ $R_{1}+R_{2}+\cdots+R_{t}$ satisfies $r-\operatorname{Bnd}(k)$, where $k=\max \left\{k_{i} \mid i=1,2, \ldots, t\right\}$.

(ii) Let $S$ be a multiplicatively closed set of central nondivisors of zero of the ring $R$. If $R$ satisfies $r-\operatorname{Bnd}(k)$, then the localization $R_{S}$ also satisfies $r-\operatorname{Bnd}(k)$.

Proof. (i) is easy and (ii) follows from the fact that $I=(I \cap R) R_{S}$ for each right ideal $I$ of $R_{S}$.

LEMMA 3. Let $\mathfrak{p}$ be a prime ideal of the commutative Noetherian ring $R$. If $R / \mathfrak{p}$ satisfies Bnd then $R / \mathfrak{p}^{s}$ satisfies Bnd for all $s \in \mathrm{Z}^{+}$.

Proof. Say $R / \mathfrak{p}$ satisfies $\operatorname{Bnd}(k)$. The proof is by induction on $s$. Say $R / \mathfrak{p}^{s-1}$ satisfies $\operatorname{Bnd}\left(k_{s-1}\right)$. The $R / \mathfrak{p}$-module $\mathfrak{p}^{s-1} / \mathfrak{p}^{s}$ is generated by, say, $m$ elements. Thus any submodule of $\mathfrak{p}^{s-1} / \mathfrak{p}^{s}$ can be generated by $m \cdot k$ elements. hence any ideal of $R / \mathfrak{p}^{s}$ can be generated by $k_{s-1}+m \cdot k$ elements and $R / \mathfrak{p}^{s}$ satisfies Bnd.

The following important theorem of $O$. Forster and R. G. Swan plays a crucial role in the proof of our result.

THEOREM. Let $R$ be a commutative Noetherian ring and $S$ an $R$-algebra which is finitely generated as an $R$-module. Assume the Krull dimension of $R$ is $d$, and let $M$ be a finitely generated right $S$-module. If for each maximal ideal $\mathfrak{m}$ of $R, M_{\mathfrak{m}}$ is generated by $r$ elements over $S_{\mathfrak{m}}$, then $M$ can be generated over $S$ by $r+d$ elements.

Proof. See Swan [12].

\section{Theorem.}

THEOREM 1. Let $R \subseteq S$ be rings with $R \subseteq Z(S)$. If $S$ is a finitely generated $R$-module then $R$ satisfies Bnd if and only if $S$ satisfies $r$-Bnd.

Proof. If $R$ satisfies Bnd, then $S$ satisfies $r$-Bnd and $l$-Bnd by Lemma 1(iii), since $S$ is a finitely generated $R$-module.

Conversely, suppose $S$ satisfies $r-\operatorname{Bnd}\left(b_{0}\right)$. Since $S$ is finitely generated as an $R$ module, $R$ is Noetherian by Eisenbud's generalization of Eakin's theorem [4]. Since $S$ is right and left Noetherian, $S$ has Krull dimension at most one by Stafford's theorem [11], and thus, since $S$ is integral over $R, R$ has Krull dimension at most one, by the "Going-Up" theorem [1]. 
Let $\mathfrak{p}_{1}, \mathfrak{p}_{2}, \ldots, \mathfrak{p}_{d}$ be the minimal primes of the commutative Noetherian ring $R$. Then there exists a positive integer $s$ such that $0=\mathfrak{p}_{1}^{s} \cap \mathfrak{p}_{2}^{s} \cap \cdots \cap \mathfrak{p}_{d}^{s}$. If we can show that each $R / \mathfrak{p}_{i}$ satisfies Bnd, then, since the sequence of $R$-modules

$$
0 \rightarrow R \rightarrow R / \mathfrak{p}_{1}^{s}+\cdots+R / \mathfrak{p}_{d}^{s}
$$

is exact, the theorem will follow from Lemma 3. Let $\mathfrak{p}$ be a minimal prime of $R$. Since $S$ is integral over $R$, there exists a prime ideal $P$ of $S$ such that $P \cap R=\mathfrak{p}$. Hence $S / P$ is a finitely generated $R / \mathfrak{p}$-module, $R / \mathfrak{p} \subseteq Z(S / P)$ and $S / P$ satisfies $r$-Bnd. Thus we may assume that $S$ is a prime ring and that $R$ is a central integral domain contained in $S$.

We utilize a clever technique of Robson and Small [7] to produce an "intermediate" ring $T$ such that

(i) $R \subseteq T \subseteq S$,

(ii) There exists a nonzero ideal $A$ of $S$ such that $A \subseteq T$,

(iii) $R \subseteq T \subseteq M_{n}(R)$ for some positive integer $n$.

$T$ is manufactured as follows: Say $S=a_{1} R+a_{2} R+\cdots+a_{t} R$. Choose a maximal $R$-independent subset of the $a_{i}$. After renaming, if necessary, we may assume that $F=a_{1} R+a_{2} R+\cdots+a_{n} R$ is a free $R$-module, and $a_{j} R \cap F \neq 0$ for $j>n$. Let $B_{j}=\left\{r \in R \mid a_{j} r \in F\right\}$. Notice that $B_{j} \neq 0$ and let $B=\bigcap_{j=1}^{t} B_{j} \neq 0$. Also $S B=B S=\sum_{i=1}^{t} a_{i} B \subseteq \sum_{i=1}^{t} a_{i} B_{i} \subseteq F$. Set $A=B S$, and let $T=R+A$. Since $T F=(R+A) F \subseteq F+A \subseteq F, F$ is a $T$-module. Since $A \subseteq F$, and $A$ is an ideal of the prime ring $S$, we have that $F$ is a faithful $T$-module. Thus there is an embedding $T \rightarrow \operatorname{End}_{R}(F)=M_{n}(R)$. When this embedding is restricted to $R$ it is canonical.

Since $S$ is finitely generated as a module over a commutative ring, $S$ satisfies a polynomial identity and thus every ideal of the prime ring $S$ intersects $Z(S)$ nontrivially. (See, for example, Rowen [8].) Let $0 \neq A_{0}=A \cap Z(S) \subseteq T \cap Z(S)=D$. Since $R \subseteq D \subseteq S, S$ is a finitely generated $D$-module, and thus, as before, $D$ is a Noetherian domain whose Krull dimension is at most one. If $D$ has Krull dimension zero, we are done since $R$ would be a field. So we may assume $D$ has Krull dimension one. There are only finitely many maximal ideals of $D$ which contain $A_{0}$. Let $\mathfrak{m}$ be a maximal ideal of $D$ which contains $A_{0}$. Now $D_{\mathfrak{m}}$ is a local Noetherian domain of Krull dimension one, and thus satisfies Bnd by Cohen's theorem [3]. Since $T$ is a finitely generated module over $D, T_{m}$ satisfies $r$-Bnd $\left(b_{m}\right)$ for some positive integer $b_{\mathfrak{m}}$. Let $b_{1}=\max \left\{b_{\mathfrak{m}} \mid \mathfrak{m}\right.$ is a maximal ideal of $D$ containing $\left.A_{0}\right\}$. Thus $T_{\mathfrak{m}}$ satisfies $r$-Bnd $\left(b_{1}\right)$ for all maximal ideals $\mathfrak{m}$ of $D$ containing $A_{0}$. Next let $\mathfrak{m}$ be a maximal ideal of $D$ which does not contain $A_{0}$. Then $A_{\mathfrak{m}}=T_{\mathfrak{m}}=S_{\mathfrak{m}}$ since $1 \in A_{\mathfrak{m}}$. Since $S_{\mathfrak{m}}$ satisfies $r$-Bnd $\left(b_{0}\right)$, for each right ideal $I$ of $T, I_{\mathfrak{m}}$ can be generated as a right $S_{\mathfrak{m}}$-module by $b_{0}$ elements, and so $I_{\mathrm{m}}$ can be generated as a $T_{\mathrm{m}}$-module by $b_{0}$ elements. Thus $T_{\mathfrak{m}}$ satisfies $r$-Bnd $\left(b_{0}\right)$ for all maximal ideals $\mathfrak{m}$ of $D$ not containing $A_{0}$. Let $b=\max \left\{b_{0}, b_{1}\right\}$. For each right ideal $I$ of $T, I_{\mathrm{m}}$ can be generated as a right $T_{\mathrm{m}}$-module by $b$ elements. By the Forster-Swan theorem, $T$ satisfies $r$-Bnd $(b+1)$.

Since $R \subseteq T \subseteq M_{n}(R), M_{n}(R)$ is a finitely generated right $T$-module and thus 
$M_{n}(R)$ satisfies $r$-Bnd $(c)$, for some positive integer $c$. Let $I$ be any ideal of $R$. since

$$
\left[\begin{array}{cccc}
I & I & \cdots & I \\
0 & 0 & \cdots & 0 \\
& \cdot & \cdot & \cdot \\
0 & 0 & \cdots & 0
\end{array}\right]
$$

is a right ideal of $M_{n}(R)$, it can be generated by $c$ elements. Clearly $I$ can be generated by $c \cdot n$ elements and $R$ satisfies Bnd.

It is natural to ask whether we can relax the condition that $S$ is a finitely generated $R$-module and replace it by the weaker condition that $S$ is integral over $R \subseteq Z(S)$ or that $S$ satisfies a polynomial identity. In the last section of this paper we show that these generalizations fail.

4. Affine PI rings. A ring $S$ is said to be ring finite over $R$ if $S$ is finitely generated as an algebra over a commutative ring $R \subseteq Z(S)$. We usually denote this by $S=R\left\{a_{1}, a_{2}, \ldots, a_{d}\right\}$. If $R$ is a field then we say that $S$ is affine over $R$. The following theorems contain, as special cases, the theorem on affine PI rings mentioned in the introduction. We begin by stating a theorem of Sarraille which plays a crucial role in the proof of our result.

THEOREM. let $S$ be a semiprime ring of Krull dimension at most one which satisfies a polynomial identity. Suppose that $S$ is ring finite over $R \subseteq Z(S)$ and that $S$ is right and left Noetherian. Then $S$ is a finitely generated module over $Z(S)$.

PROOF. See Theorem 2.18 of Sarraillé [10].

THEOREM 2. Let $S=R\left\{a_{1}, a_{2}, \ldots, a_{d}\right\}$ be ring finite over $R \subseteq Z(S)$. Suppose that $S$ is semiprime and satisfies a polynomial identity. If $S$ satisfies $r$-Bnd, then $S$ also satisfies $l$-Bnd.

ProOF. $S$ is right Noetherian and hence it is left Noetherian by Cauchon's theorem [2]. Stafford's theorem [11] says that $S$ has Krull dimension at most one. An application of Sarraille's theorem yields that $S$ is module finite over its center $Z=Z(S)$. Theorem 1 says that $Z$ satisfies Bnd and thus $S$ satisfies $l$-Bnd.

By using standard ring-theoretic techniques it is now possible to replace the semiprime hypothesis in Theorem 2 by assuming the weaker condition that $S$ is Noetherian on both sides.

THEOREM 3. Let $S=R\left\{a_{1}, a_{2}, \ldots, a_{d}\right\}$ be ring finite over $R \subseteq Z(S)$. Suppose that $S$ is right and left Noetherian and satisfies a polynomial identity. If $S$ satisfies $r$-Bnd, then $S$ also satisfies l-Bnd.

PROOF. Let $N$ be the prime radical of $S$, and let $t$ be a positive integer such that $N^{t}=0$. By Theorem $2, \bar{S}=S / N$ satisfies $l$-Bnd. For $i=1,2, \ldots, t-1$ we have that $N^{i} / N^{i+1}$ is a finitely generated left $\bar{S}$-module, and hence $N^{i} / N^{i+1}$ satisfies $l$-Bnd as a left $\bar{S}$-module and thus as a left $S$-module. Since $\left(N^{i} / N^{i+2}\right) /\left(N^{i+1} / N^{i+2}\right) \simeq$ $N^{i} / N^{i+1}$, repeated application of Lemma 1(ii) implies that $N^{i} / N^{i+j}$ satisfies $l$-Bnd as an $S$-module for $j \geq 1$. In particular, $N \simeq N / N^{t}$ satisfies $l$-Bnd as an $S$-module. Since $N$ and $S / N$ satisfy $l$-Bnd, we have that $S$ satisfies $l$-Bnd. 
5. Examples. The first example is due to Wadsworth and Schelter (see Sarraillé [10]). We will construct a prime ring $S$ which satisfies both $r$-Bnd and $l$-Bnd as well as a polynomial identity, but for which $Z=Z(S)$ does not satisfy Bnd. This and the next example show that the natural generalizations of Theorem 1 fail.

EXAMPLE 1. Let $F_{1}$ and $F_{2}$ be subfields of the field $E$ such that $E$ is a finite extension of $F_{1}$ and $F_{2}$, but $E$ is an infinite extension of $F=F_{1} \cap F_{2}$. Set

$$
S=\left[\begin{array}{cc}
F_{1}+x E[x] & x E[x] \\
x E[x] & F_{2}+x E[x]
\end{array}\right]
$$

and

$$
R=\left\{\left[\begin{array}{cc}
f(x) & 0 \\
0 & f(x)
\end{array}\right] \mid f(x) \in F+x E[x]\right\},
$$

where $x$ is an indeterminate. Since $M_{2}(x E[x])$ is an ideal of both $S$ and $M_{2}(E[x])$, and since $M_{2}(E[x])$ is a prime ring, we have that $S$ is a prime ring. Since $S \subseteq$ $M_{2}(E[x]), S$ satisfies a polynomial identity. For $i=1,2, F_{i}+x E[x]$ satisfies Bnd since $E[x]$ is a finitely generated module over $F_{i}+x E[x]$. Thus

$$
T=\left[\begin{array}{cc}
F_{1}+x E[x] & 0 \\
0 & F_{2}+x E[x]
\end{array}\right] \text { satisfies Bnd. }
$$

Since $S$ is finitely generated as a right and left module over $T, S$ satisfies $r$-Bnd and $l$-Bnd. Finally, $R=Z(S)$ and $R$ does not satisfy Bnd since $R$ is not even Noetherian.

Our next example is a ring $S$ which satisfies $r$-Bnd and $l$-Bnd and is integral over its center $R$ which is not Noetherian. This example is taken from Blair [1] and is based on a commutative ring constructed by Mollier [6].

EXAMPLE 2. Mollier has constructed a commutative ring $B=k[T] /\left(T^{2}\right)$ where $k$ is a field of characteristic two such that the degree of $k$ over $k^{2}$, the subfield of squares, is infinite. $B$ is equipped with an automorphism $\sigma$ of order two such that $B^{\sigma}=\{b \in B \mid \sigma(b)=b\}=k^{2}+k t$, where $t=T+\left(T^{2}\right)$, is a non-Noetherian subring of $B$. Also note that $B$ is a two-dimensional algebra over the field $k$.

Form the skew polynomial ring $B[X, \sigma]$ where multiplication is given by $X b=$ $\sigma(b) X$ for $b \in B$. Let $S=B[X, \sigma] /\left(X^{2}\right)$ and let $x$ be the image of $X$ modulo $\left(X^{2}\right) . S$ is a finitely generated right and left module over $B$ and hence over $k$. Thus $S$ satisfies $r$-Bnd and $l$-Bnd. Let $A=\{b \in B \mid b c=b \sigma(c)$ for all $c \in B\}$. Then $R=Z(S)=\left\{b_{0}+b_{1} x \mid b_{0} \in B^{\sigma}, b_{1} \in A\right\}$. If $I$ is an ideal of $B^{\sigma}$ then $I^{*}=$ $\left\{b_{0}+b_{1} x \mid b_{0} \in I, b_{1} \in A\right\}$ is an ideal of $R$, and so $R$ is not Noetherian and hence does not satisfy Bnd. If $s=b_{0}+b_{1} x \in S$, then $s$ satisfies $X^{2}-\left(b_{0}+\sigma\left(b_{0}\right)\right) X+b_{0} \sigma\left(b_{0}\right)=0$, while $b_{0}+\sigma\left(b_{0}\right)$ and $b_{0} \sigma\left(b_{0}\right)$ are elements of $B^{\sigma} \subseteq R$. Finally, one can also check that $S$ satisfies the standard identity of degree 4 .

The next example is an affine PI ring which satisfies $r$-Bnd but not $l$-Bnd. This shows that the hypotheses of Theorems 2 and 3 are needed.

EXAMPLE 3. Let $F$ be a field and set

$$
S=\left[\begin{array}{ll}
F & F[x] \\
0 & F[x]
\end{array}\right]
$$


where $x$ is an indeterminate. Clearly

$$
T=\left[\begin{array}{cc}
F & 0 \\
0 & F[x]
\end{array}\right]
$$

satisfies Bnd and since $S$ is a finitely generated right $T$-module, $S$ satisfies $r$-Bnd. On the other hand $S$ fails to satisfy $l$-Bnd since it is not left Noetherian.

\section{REFERENCES}

1. W. D. Blair, Right Noetherian rings integral over their centers, J. Algebra 27 (1973), 187-198.

2. G. Cauchon, Anneaux semi-premiers, noethériens, à identitiés polynomiales, Bull. Soc. Math. France 104 (1976), 99-111.

3. I. S. Cohen, Commutative rings with restricted minimum condition, Duke Math. J. 17 (1950), 27-42.

4. D. Eisenbud, Subrings of Artinian and Noetherian rings, Math. Ann. 185 (1970), 247-249.

5. A. V. Jategaonkar, A counterexample in ring theory and homological algebra, J. Algebra 12 (1969), 418-440.

6. D. Mollier, Descente de la propriété noethérienne, Bull. Sci. Math. 94 (1970), 25-31.

7. J. C. Robson and L. W. Small, Liberal extensions, Proc. London Math. Soc. (3) 42 (1981), 87-103.

8. L. H. Rowen, Polynomial identities in ring theory, Academic Press, New York, 1980.

9. J. D. Sally, Some results on multiplicity with applications to bounded and two dimensional prime bounded rings, J. Algebra 35 (1975), 224-234.

10. J. J. Sarraillé, Module finiteness of low-dimensional PI rings, Pacific J. Math. 102 (1982), 189-208.

11. J. T. Stafford, Rings with a bounded number of generators for right ideals, Quart. J. Math. Oxford Ser. (2) 34 (1983), 107-114.

12. R. G. Swan, The number of generators of a module, Math. Z. 102 (1967), 318-322.

Department of Mathematical Sciences, Northern Illinois University, De KALB, ILLINOIS 60115 